\title{
The Birman-Craggs-Johnson homomorphism and abelian cycles in the Torelli group
}

\author{
Tara E. Brendle and Benson Farb *
}

November 19, 2018

\begin{abstract}
In the 1970's, Birman-Craggs-Johnson [BC, Jo1] used Rochlin's invariant for homology 3-spheres to construct a remarkable surjective homomorphism $\sigma: \mathcal{I}_{\mathrm{g}, 1} \rightarrow B_{3}$, where $\mathcal{I}_{\mathrm{g}, 1}$ is the Torelli group and $B_{3}$ is a certain $\mathbf{F}_{2}$-vector space of Boolean (square-free) polynomials. By pulling back cohomology classes and evaluating them on abelian cycles, we construct $16 g^{4}+O\left(g^{3}\right)$ dimensions worth of nontrivial elements of $H^{2}\left(\mathcal{I}_{\mathrm{g}, 1}, \mathbf{F}_{2}\right)$ which cannot be detected rationally. These classes in fact restrict to nontrivial classes in the cohomology of the subgroup $\mathcal{K}_{\mathrm{g}, 1}<\mathcal{I}_{\mathrm{g}, 1}$ generated by Dehn twists about separating curves.

We also use the "Casson-Morita algebra" and Morita's integral lift of the BirmanCraggs-Johnson map restricted to $\mathcal{K}_{\mathrm{g}, 1}$ to give the same lower bound on $H^{2}\left(\mathcal{K}_{\mathrm{g}, 1}, \mathbf{Z}\right)$.
\end{abstract}

\section{Contents}

1 Introduction 2

2 The Birman-Craggs-Johnson homomorphism 5

2.1 Birman-Craggs homomorphisms and Sp-quadratic forms . . . . . . . . . . 5

2.2 Johnson's formula for $\sigma \ldots \ldots \ldots \ldots \ldots \ldots$

3 Abelian cycles in $H_{2}\left(\mathcal{K}_{\mathrm{g}, 1}, \mathbf{F}_{2}\right) \quad 7$

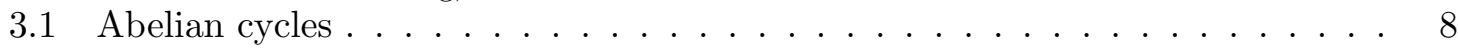

3.2 Calculations with $\sigma_{*} \ldots \ldots \ldots \ldots \ldots \ldots$

4 Integral abelian cycles and the Casson-Morita algebra 13

4.1 The Casson-Morita algebra . . . . . . . . . . . . . . . . 13

4.2 Morita's homomorphism $\rho \ldots \ldots \ldots \ldots \ldots$

4.3 Lifting abelian cycles . . . . . . . . . . . . . . . 16

5 Cohomology 17

*The first author is partially supported by NSF grant DMS-0504208 and was also supported in part by a VIGRE postdoc under NSF grant number 9983660 to Cornell University. The second author is supported in part by NSF grant DMS-0244542. 


\section{Introduction}

Let $\Sigma_{g, 1}$ denote the compact, oriented surface of genus $g$ with one boundary component. The mapping class group $\operatorname{Mod}_{g, 1}$ is the group of isotopy classes of orientation-preserving self-homeomorphisms $\Sigma_{g, 1}$, where both the homeomorphisms and the isotopies are taken to fix the boundary $\partial \Sigma_{g, 1}$ pointwise. While versions of our results extend to closed surfaces, we consider this case because it is somewhat simpler technically.

Algebraic intersection number gives a symplectic form on $H_{1}\left(\Sigma_{g, 1}, \mathbf{Z}\right)$. This form is preserved by the natural action of $\operatorname{Mod}_{g, 1}$. The Torelli group $\mathcal{I}_{\mathrm{g}, 1}$ is defined to be the kernel of this action. We then have an exact sequence

$$
1 \rightarrow \mathcal{I}_{\mathrm{g}, 1} \rightarrow \operatorname{Mod}_{g, 1} \rightarrow \mathrm{Sp}(2 g, \mathbf{Z}) \rightarrow 1
$$

We will also consider the bounding twist group $\mathcal{K}_{\mathrm{g}, 1}$, which is the subgroup of $\mathcal{I}_{\mathrm{g}, 1}$ generated by Dehn twists about those curves which separate $\Sigma_{g, 1}$. Johnson found a homomorphism $\tau$ and proved that it gives the following exact sequence (see [Jo3, Jo5, Jo6]):

$$
1 \rightarrow \mathcal{K}_{\mathrm{g}, 1} \rightarrow \mathcal{I}_{\mathrm{g}, 1} \stackrel{\tau}{\rightarrow} \wedge^{3} H \rightarrow 1
$$

where $H=H_{1}\left(\Sigma_{g, 1} ; \mathbf{Z}\right)$.

While there has been spectacular progress in understanding $H^{*}\left(\operatorname{Mod}_{g, 1}, \mathbf{Z}\right)$ (see $\left.[\mathrm{MW}]\right)$, very little is known about $H^{*}\left(\mathcal{I}_{\mathrm{g}, 1}, \mathbf{Z}\right)$, and even less is known about $H^{*}\left(\mathcal{K}_{\mathrm{g}, 1}, \mathbf{Z}\right)$. For example, although it was recently shown in $[\mathrm{BF}]$ that $\mathcal{K}_{\mathrm{g}, 1}$ is infinitely generated, it is still not known whether or not $H^{1}\left(\mathcal{K}_{\mathrm{g}, 1}, \mathbf{Z}\right)$ is finitely generated. Note that, as follows from (1), for any field $K$ the vector space $H^{*}\left(\mathcal{I}_{\mathrm{g}, 1}, K\right)$ is a module over $\operatorname{Sp}(2 g, K)$.

Akita $[\mathrm{Ak}]$ has shown that the algebras $H^{*}\left(\mathcal{I}_{\mathrm{g}}, \mathbf{Q}\right)$ and $H^{*}\left(\mathcal{K}_{\mathrm{g}}, \mathbf{Q}\right)$ must be infinite dimensional for $g \geq 7$, although these groups vanish in sufficiently high degrees. His proof is by contradiction, however, and produces no explicit classes. One goal is, therefore, to construct explicit classes. Morita [Mo2] has identified a certain ( $\operatorname{Mod}_{g, 1}$-invariant) secondary

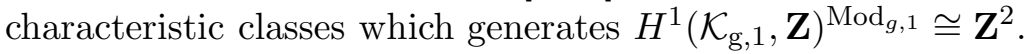

In a series of papers Johnson proved the difficult result:

$$
H_{1}\left(\mathcal{I}_{\mathrm{g}, 1}, \mathbf{Z}\right) \approx \wedge^{3} H \oplus B_{2}
$$

where $B_{2}$ consists of 2-torsion; see [Jo4], [Jo2] for a summary, or [vdB]. While the $\wedge^{3} H$ piece comes from purely algebraic considerations, the $B_{2}$ piece is "deeper" in the sense that it is purely topological, and comes from the Rochlin invariant (see below); indeed the former appears in $H_{1}$ of the "Torelli group" in the analogous theory for $\operatorname{Out}\left(F_{n}\right)$, while the latter does not.

Problem: Determine the subalgebras of $H^{*}\left(\mathcal{I}_{\mathrm{g}, 1}, K\right)$, for $K=\mathbf{Q}$ and $K=\mathbf{F}_{2}$, generated by $H^{1}\left(\mathcal{I}_{\mathrm{g}, 1}, \mathbf{Z}\right) \otimes K$.

Rational cohomology. Johnson proved in [Jo4] that the induced map in cohomology

$$
\tau^{*}: H^{*}\left(\wedge^{3} H, \mathbf{Q}\right) \rightarrow H^{*}\left(\mathcal{I}_{\mathrm{g}, 1}, \mathbf{Q}\right)
$$


is an isomorphism in degree one. Hain [Hai] determined the image (and kernel) of $\tau^{*}$ in degree two, as did Sakasai [Sa] (up to a small unknown) in degree three.

A key tool in the proofs of these results is the fact that $\tau^{*}$ is a map of $\operatorname{Sp}(2 g, \mathbf{Q})$-modules. Since $H^{*}\left(\wedge^{3} H, \mathbf{Q}\right)$ decomposes as a direct sum of easily described irreducible representations, the calculation of $\tau^{*}$ is greatly simplified. Note that since $\mathcal{K}_{\mathrm{g}, 1}$ lies in (indeed equals) the kernel of $\tau$, this method yields no information about $\mathcal{K}_{\mathrm{g}, 1}$.

The Birman-Craggs-Johnson homomorphism. Consider a fixed Heegaard embedding $h: \Sigma_{g} \hookrightarrow S^{3}$ of the closed surface $\Sigma_{g}$. An element of $f \in \mathcal{I}_{\mathrm{g}}$ then determines an integral homology 3 -sphere $M_{f}$ by cutting out a handlebody determined by $h$ and gluing it back in via $f$. Birman-Craggs $[\mathrm{BC}]$ proved that the map $f \mapsto \mu\left(M_{f}\right)$ taking $f$ to the Rochlin invariant $\mu$ of $M_{f}$ actually determines a homomorphism $\mathcal{I}_{\mathrm{g}} \rightarrow \mathbf{F}_{2}$. The is called the Birman-Craggs homomorphism corresponding to $h$.

Building on their work, Johnson constructed in [Jo1] the surjective Birman-CraggsJohnson (BCJ) homomorphism $\sigma: \mathcal{I}_{\mathrm{g}, 1} \rightarrow B_{3}$, where $B_{3}$ is the degree $\leq 3$ summand of a certain graded $\mathbf{F}_{2}$-algebra $B$ of "Boolean polynomials" in $2 g$ variables (see Section 2.1 below). The map $\sigma$ encodes all of the Birman-Craggs homomorphisms in the sense that the kernel of $\sigma$ is the intersection of the kernels of all Birman-Craggs homomorphisms. As with Johnson's homomorphism $\tau$, the group $\operatorname{Mod}_{g, 1}$ acts naturally on both $\mathcal{I}_{\mathrm{g}, 1}$ and on $B_{3}$, and $\sigma$ respects this action.

Johnson showed that, unlike the case of the map $\tau$, the restriction of $\sigma$ to $\mathcal{K}_{\mathrm{g}, 1}$ is highly nontrivial. In fact, he showed that:

$$
\left.\sigma\right|_{\mathcal{K}_{\mathrm{g}, 1}}: \mathcal{K}_{\mathrm{g}, 1} \rightarrow B_{2}
$$

is a surjection onto the degree $\leq 2$ summand $B_{2}$ of $B$. Further, he showed that the $B_{3}-$ $B_{2}$ piece of image $(\sigma)$ is precisely image $(\tau)=\wedge^{3} H$ reduced mod 2. For this reason we concentrate here on the "truly 2 -torsion" summand $B_{2}$. Our main result is the following.

Theorem 1. Each of the images of

$$
\begin{gathered}
\sigma^{*}: H^{2}\left(B_{2}, \mathbf{F}_{2}\right) \rightarrow H^{2}\left(\mathcal{I}_{\mathrm{g}, 1}, \mathbf{F}_{2}\right) \\
\left(\left.\sigma\right|_{\mathcal{K}_{\mathrm{g}, 1}}\right)^{*}: H^{2}\left(B_{2}, \mathbf{F}_{2}\right) \rightarrow H^{2}\left(\mathcal{K}_{\mathrm{g}, 1}, \mathbf{F}_{2}\right)
\end{gathered}
$$

have dimension at least $16 g^{4}+O\left(g^{3}\right)$. Further, this lower bound is correct to third order: the subspaces of cocycles we describe below have codimension at most $4 g^{2}$ in the images of $\sigma^{*}\left(\operatorname{resp} .\left(\left.\sigma\right|_{\mathcal{K}_{\mathrm{g}, 1}}\right)^{*}\right)$.

Note that none of these classes can be detected via the Johnson homomorphism $\tau$. Another aspect of this problem which interested us is the failure of representation theory in this context. While $\sigma^{*}: H^{*}\left(B_{2}, \mathbf{F}_{2}\right) \rightarrow H^{*}\left(\mathcal{I}_{\mathrm{g}, 1}, \mathbf{F}_{2}\right)$ is a homomorphism of $\operatorname{Sp}\left(2 g, \mathbf{F}_{2}\right)$-algebras, the module $H^{*}\left(B_{2}, \mathbf{F}_{2}\right)$ no longer decomposes as a direct sum of irreducible representations. Further, the seemingly simple (characteristic two) modular representation theory needed to aid in computations (as in the rational case) seems to be beyond what is currently known. Thus we are forced to use more involved topological methods for computations. 
The Casson-Morita algebra. Much, but not all, of the situation for the BCJ homomorphism described above can be lifted to $\mathbf{Z}$ coefficients. Replacing the Rochlin invariant $\mu$ with the Casson invariant $\lambda$, one can consider the map from the Torelli group of the closed surface $\mathcal{I}_{\mathrm{g}} \rightarrow \mathbf{Z}$ given by $f \mapsto \lambda\left(M_{f}\right)$. While this map is definitely not a homomorphism, Morita proved in [Mo1] that it is a homomorphism when restricted to $\mathcal{K}_{\mathrm{g}}$. Varying over Heegaard embeddings $\Sigma_{g} \hookrightarrow S^{3}$ gives different homomorphisms. Morita put these together (passing for technical reasons to the case of one boundary component) to give a homomorphism $\rho: \mathcal{K}_{\mathrm{g}, 1} \rightarrow \mathcal{A}$ for a certain $\mathbf{Z}$-algebra $\mathcal{A}$ which we call the Casson-Morita algebra, which is a kind of universal receptor for Casson invariants. This lifts the BCJ picture as follows (see Proposition 5 below): there exists a homomorphism $\mu: \mathcal{A} \rightarrow B_{2}$ such that the following diagram commutes:

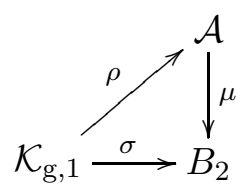

We can then use the homomorphism

$$
\rho^{*}: H^{*}(\mathcal{A}, \mathbf{Z}) \rightarrow H^{*}\left(\mathcal{K}_{\mathrm{g}, 1}, \mathbf{Z}\right)
$$

to obtain nontrivial classes in $H^{*}\left(\mathcal{K}_{\mathrm{g}, 1}, \mathbf{Z}\right)$. An easy argument shows that our computations in the $\mathbf{F}_{2}$ case imply the following.

Corollary 2. The image of $\rho^{*}$ in $H^{2}\left(\mathcal{K}_{\mathrm{g}, 1}, \mathbf{Z}\right)$ contains an $\operatorname{Sp}(2 g, \mathbf{Z})$-submodule of rank $16 g^{4}+O\left(g^{3}\right)$.

We remark that, apart from allowing us to use $\sigma^{*}$ (which is defined on all of $\mathcal{I}_{\mathrm{g}, 1}$ ), the use of $\mathbf{F}_{2}$ coefficients greatly simplifies computations. For these reasons, together with our desire for an explicit topological construction of cycles, we decided not to work with Morita's homomorphism $\rho$ (and with $Z$ coefficients) directly.

Abelian cycles. A pair of commuting elements $f, g$ in a group $\Gamma$ determines a homomorphism $i: \mathbf{Z}^{2} \rightarrow \Gamma$. The abelian cycle $\{f, g\} \in H_{2}(\Gamma, \mathbf{Z})$ determined by the pair $f, g$ is the image of the fundamental class under the homomorphism $H_{2}\left(\mathbf{Z}^{2}, \mathbf{Z}\right) \rightarrow H_{2}(\Gamma, \mathbf{Z})$. While Theorem 1 is stated for convenience in cohomology, we mostly study homology, since our method for proving that the cohomology classes in the image of $\sigma^{*}$ are nontrivial is to evaluate these classes on abelian cycles; see $\S 3$ below.

Acknowledgements. We thank Nathan Broaddus, Ken Brown, Allen Hatcher, Shigeyuki Morita and Peter Sin for their valuable expertise and comments. We are grateful to Vijay Ravikumar for his useful insights regarding "index-matched" elements and for working out the case of bounding pair maps while participating in Cornell University's REU program in 2005 under the supervision of the first author. We also wish to thank the other REU students, Tova Brown, Tom Church, Peter Maceli, and Aaron Pixton, for helpful discussions. 


\section{The Birman-Craggs-Johnson homomorphism}

We begin with a brief review of the work of Birman-Craggs and of Johnson relating the Rochlin invariant of 3-manifolds to certain algebraic structures associated to surfaces and their Torelli groups.

\subsection{Birman-Craggs homomorphisms and Sp-quadratic forms}

Let $M$ be an oriented, integral homology 3 -sphere endowed with a spin structure. Choose $X$, a spin 4-manifold such that $\partial X=M$ and such that this restriction to the boundary induces the given spin structure on $M$. The Rochlin invariant $\mu(M) \in \mathbf{F}_{2}$ of $M$ is given by

$$
\mu(M):=\frac{\sigma(X)}{8} \bmod 2
$$

where $\sigma(X)$ denotes the signature of $X$; it does not depend on the choice of the 4-manifold $X$ (see, e.g. [GS]). Note that $\mu\left(S^{3}\right)=0$.

We give the definition of the Birman-Craggs homomorphisms as reformulated by Johnson for the case of a surface with one boundary component [Jo5]. A Heegaard embedding of the surface $\Sigma_{g, 1}$ in $S^{3}$ is an embedding $h: \Sigma_{g, 1} \rightarrow S^{3}$ such that $h\left(\Sigma_{g, 1}\right) \subset S$, where $S$ is a Heegaard surface for $S^{3}$. Let $h$ be such a Heegaard embedding, and let $f \in \mathcal{I}_{\mathrm{g}}$. Now split $S^{3}$ along $h\left(\Sigma_{g, 1}\right)$ and reglue via the map $f$. Specifically, we map a point $x$ in one copy of the interior of $h\left(\Sigma_{g, 1}\right)$ to $h f h^{-1}(x)$ in the other copy. The resulting 3-manifold $M(h, f)$ is necessarily a homology 3-sphere. We can therefore define the Birman-Craggs homomorphism $\rho_{h}: \mathcal{I}_{\mathrm{g}, 1} \rightarrow \mathbf{Z}_{2}$ associated to the Heegaard embedding $h$ via the formula

$$
\rho_{h}(f):=\mu(M(h, f))
$$

Self-linking forms. Given a Heegaard embedding $h$ of the oriented surface $\Sigma_{g, 1}$ in $S^{3}$, there is a corresponding mod 2 self-linking form $\omega_{h}: H_{1}\left(\Sigma_{g, 1}, \mathbf{Z}\right) \rightarrow \mathbf{F}_{2}$ defined on irreducible elements $c \in H_{1}\left(\Sigma_{g, 1}, \mathbf{Z}\right)$ via $\omega_{h}(c)=l k\left(h(c), h(c)^{+}\right)$; here the latter expression denotes the linking number of a representative of the homology class $h(c)$ with its positive push-off $h(c)^{+}$ in $S^{3}$. For simplicity, we let $H=H_{1}\left(\Sigma_{g, 1}, \mathbf{F}_{2}\right)$.

Johnson showed that all such mod 2 self-linking forms arise as functions $\omega: H \rightarrow \mathbf{F}_{2}$

whose associated bilinear form is just the usual symplectic intersection pairing on $H$, and vice-versa. In other words, if $\Omega$ denotes the set of all mod 2 self-linking forms, then we have

$$
\Omega=\left\{\omega: H \rightarrow \mathbf{F}_{2} \mid \omega(a+b)=\omega(a)+\omega(b)+a \cdot b\right\}
$$

where $a \cdot b$ denotes the intersection form. In particular, given any $\omega \in \Omega$, we have $\omega=\omega_{h}$ for some Heegaard embedding $h$. Moreover, Johnson showed that the value of the BirmanCraggs homomorphism $\rho_{h}(f)$ is completely determined by the self-linking form $\omega_{h}$ and by $f \in \mathcal{I}_{\mathrm{g}, 1}[\mathrm{Jo} 1]$. 
The BCJ map. Johnson is then able to combine all Birman-Craggs homomorphisms into a single, surjective map $\sigma$ from $\mathcal{I}_{\mathrm{g}, 1}$ into the vector space of functions $\Omega \rightarrow \mathbf{F}_{2}$, defined as follows:

$$
\sigma(f)(\omega)=\rho_{h}(f)=\mu(h, f)
$$

where $\omega=\omega_{h}$, as above. We call $\sigma$ the Birman-Craggs-Johnson homomorphism, or BCJ homomorphism for short. It is clear that $\operatorname{ker} \sigma=\cap_{h} \operatorname{ker} \rho_{h}$, where the intersection is taken over all Birman-Craggs homomorphisms.

\subsection{Johnson's formula for $\sigma$}

Let $a_{1}, b_{1}, \ldots, a_{g}, b_{g}$ be a fixed symplectic basis for $H$. Now for each $c \in H$, we can define a map $\bar{c}: \Omega \rightarrow \mathbf{F}_{2}$ via $\omega \mapsto \omega(c)$. We will need two basic facts which follow directly from the definitions:

$$
\begin{gathered}
\overline{a+b}=\bar{a}+\bar{b}+a \cdot b \text { for all } a, b \in H \\
\bar{c}^{2}=\bar{c} \text { for all } c \in H
\end{gathered}
$$

The "square-free" condition in (5) gives the space of such elements a particularly nice structure, which we now describe.

Boolean polynomials. We define $B\left(x_{1}, \ldots, x_{n}\right)$, the ring of Boolean (or square-free) polynomials, to be the quotient of the usual polynomial ring on the variables $x_{1}, \ldots, x_{n}$ with coefficients in $\mathbf{F}_{2}$ by the ideal generated by the relations $x_{i}^{2}=x_{i}$ for all $i=1, \ldots, n$. In other words

$$
B\left(x_{1}, \ldots, x_{n}\right)=\mathbf{F}_{2}\left[x_{1}, \ldots, x_{n}\right] /<x_{i}^{2}=x_{i}>
$$

Then we let $B_{r}=B_{r}\left(x_{1}, \ldots, x_{n}\right)$ denote the subset of $B\left(x_{1}, \ldots, x_{n}\right)$ consisting of those polynomials of degree at most $r$. For our purposes, we will take $n=2 g$, and we will attach a certain topological significance to these $2 g$ variables.

We form Boolean polynomials in the obvious way out of elements $\bar{c}$, with $c \in H$. Specifically, we take as abstract variables the $2 g$ maps $\bar{a}_{1}, \ldots, \bar{a}_{g}, \bar{b}_{1}, \ldots \bar{b}_{g}$. As above, we denote the ring of Boolean polynomials of degree at most $r$ in these $2 g$ variables simply by $B_{r}$. A basis for $B_{2}$ as a vector space over $\mathbf{F}_{2}$ consists of the $2 g^{2}+g+1$ elements

$$
\left\{1, \bar{a}_{i}, \bar{b}_{i}, \bar{a}_{i} \bar{b}_{j}, \bar{a}_{i} \bar{a}_{j}(i \neq j), \bar{b}_{i} \bar{b}_{j}(i \neq j)\right\} .
$$

Johnson proves in [Jo1] that the image of $\sigma$ in the vector space of functions $\Omega \rightarrow \mathbf{F}_{2}$ is in fact isomorphic to $B_{3}$. Thus we can write

$$
\sigma: \mathcal{I}_{\mathrm{g}, 1} \rightarrow B_{3}
$$

Explicit formulas on generators. Recall that twists about separating curves generate $\mathcal{K}_{\mathrm{g}, 1}$ by definition. In $\S 7$ of [Jo1], Johnson gives an explicit formula for $\sigma\left(T_{\gamma}\right)$ when $\gamma$ is a separating curve on $\Sigma_{g, 1}$. The curve $\gamma$ separates $\Sigma_{g, 1}$ into two components; let $\Sigma^{\prime}$ denote 
the component which does not contain $\partial \Sigma_{g, 1}$. Let $A_{1}, B_{1}, \ldots, A_{g\left(\Sigma^{\prime}\right)}, B_{g\left(\Sigma^{\prime}\right)}$ be a symplectic $Z_{2}$-homology basis for $\Sigma^{\prime}$. Then Johnson's formula is:

$$
\sigma\left(T_{\gamma}\right)=\sum_{i=1}^{g\left(\Sigma_{\alpha}\right)} \bar{A}_{i} \bar{B}_{i}
$$

The expression on the right-hand side of the equation is independent of the choice of symplectic basis for $\Sigma_{\gamma \delta}$. The restriction $\left.\sigma\right|_{\mathcal{K}_{\mathrm{g}, 1}}$ is surjective onto $B_{2} \subset B_{3}$.

Johnson gives a similar formula for generators of $\mathcal{I}_{\mathrm{g}, 1}$. We will not need this formula in our calculations, but we give it here for the sake of completeness. Recall that a bounding pair map (BP map for short) in $\mathcal{I}_{\mathrm{g}, 1}$ is the composition $T_{\gamma} T_{\delta}^{-1}$ of Dehn twists about nonseparating simple closed curves $\gamma, \delta$ whose union $\gamma \cup \delta$ separates $\Sigma_{g, 1}$. Given a BP map $T_{\gamma} T_{\delta}^{-1}$, we let $\Sigma^{\prime}$ be the component of $\Sigma_{g, 1} \backslash(\gamma \cup \delta)$ which does not contain $\partial \Sigma_{g, 1}$, as above. Let $C$ be the homology class of $\gamma$. Then Johnson's formula for $\sigma: \mathcal{I}_{\mathrm{g}, 1} \rightarrow B_{3}$ is as follows:

$$
\sigma\left(T_{\gamma} T_{\delta}^{-1}\right)=\left(\sum_{i=1}^{g\left(\Sigma^{\prime}\right)} \bar{A}_{i} \bar{B}_{i}\right)(\bar{C}+1) \in B_{3}
$$

Since BP maps generate $\mathcal{I}_{\mathrm{g}, 1}$ (see [Jo2]), this formula can be taken as the definition of the $\operatorname{map} \sigma$.

Remark. As previously noted, $B_{3}$ is a $\operatorname{Mod}_{\mathrm{g}, 1}$-module in a fairly obvious way. We have that $f \in S p\left(2 g, \mathbf{Z}_{2}\right)$ acts on a map $\omega: H \rightarrow \mathbf{Z}_{2}$ in $\Omega$ by $f \cdot \omega(x)=\omega(f(x))$. Then $S p$ acts on a function $\phi: \Omega \rightarrow \mathbf{Z}_{2}$ adjoint to its action on $\Omega$, i.e., $f \cdot \phi(\omega)=\phi(f \cdot \omega)$. Furthermore, $\sigma$ is a $\operatorname{Mod}_{\mathrm{g}, 1}$-equivariant map; that is, for $f \in \operatorname{Mod}_{g, 1} h \in \mathcal{I}_{\mathrm{g}, 1}$ we have $\sigma\left(f h f^{-1}\right)=\hat{f} \cdot \sigma(h)$, where $\hat{f}$ denotes the image of the map $f$ under the symplectic representation mod 2. Moreover, since $\mathcal{K}_{\mathrm{g}, 1}$ is a $\operatorname{Mod}_{\mathrm{g}, 1}$-submodule of $\mathcal{I}_{\mathrm{g}}$, we have that $\sigma\left(\mathcal{K}_{\mathrm{g}, 1}\right)=B_{2}$ is also a $\operatorname{Mod}_{\mathrm{g}, 1}$-module and that $\left.\sigma\right|_{\mathcal{K}_{\mathrm{g}, 1}}$ is also a $\operatorname{Mod}_{\mathrm{g}, 1}$-equivariant map.

Henceforth we will restrict our attention to the subgroup $\mathcal{K}_{\mathrm{g}, 1}$ in $\mathcal{I}_{\mathrm{g}, 1}$ and write simply $\sigma: \mathcal{K}_{\mathrm{g}, 1} \rightarrow B_{2}$ for the BCJ homomorphism. However, the tools and techniques we are about to discuss will extend to the case of the full Torelli group.

\section{Abelian cycles in $H_{2}\left(\mathcal{K}_{\mathrm{g}, 1}, \mathbf{F}_{2}\right)$}

In this section, we will give a method for constructing nontrivial classes in $H_{2}\left(\mathcal{K}_{\mathrm{g}, 1}, \mathbf{F}_{2}\right)$, inspired by Sakasai [Sa]. The idea is to construct abelian cycles in $H_{2}\left(\mathcal{K}_{\mathrm{g}, 1}, \mathbf{F}_{2}\right)$ and then to show that their images under the induced map

$$
\sigma_{*}: H_{2}\left(\mathcal{K}_{\mathrm{g}, 1}, \mathbf{F}_{2}\right) \rightarrow H_{2}\left(B_{2}, \mathbf{F}_{2}\right) \cong \wedge^{2} B_{2} \oplus B_{2}
$$

are nontrivial. 


\subsection{Abelian cycles}

If $f, g \in \mathcal{K}_{\mathrm{g}, 1}$ commute then we have a homomorphism $i: \mathbf{Z}^{2} \rightarrow \mathcal{K}_{\mathrm{g}, 1}$, which induces a homomorphism

$$
i_{*}: H_{2}\left(\mathbf{Z}^{2}, \mathbf{F}_{2}\right) \rightarrow H_{2}\left(\mathcal{K}_{\mathrm{g}, 1}, \mathbf{F}_{2}\right)
$$

The image of the generator $t$ of $H_{2}\left(\mathbf{Z}^{2}, \mathbf{F}_{2}\right)$ is denoted by

$$
\{f, g\}:=i_{*}(t) \in H_{2}\left(\mathcal{K}_{\mathrm{g}, 1}, \mathbf{F}_{2}\right)
$$

The homology class $\{f, g\}$ is called the abelian cycle corresponding to the pair $f, g$.

While we have given the definition of abelian cycles for the case of $H_{2}\left(\mathcal{K}_{\mathrm{g}, 1}, \mathbf{F}_{2}\right)$, one can generalize the construction of abelian cycles in the obvious way for different choices of groups and of coefficients, and also for degree $n>2$ by taking $n$ commuting elements and looking at the injection $\mathbf{Z}^{n} \hookrightarrow \mathcal{K}_{\mathrm{g}, 1}$.

Homology of $\boldsymbol{B}_{\mathbf{2}}$. Recall that as a vector space over $\mathbf{F}_{2}$, the summand $B_{2}$ of the algebra $B$ is spanned by $d=2 g^{2}+g+1$ Boolean monomials. Thus

$$
H_{2}\left(B_{2}, \mathbf{F}_{2}\right) \cong \wedge^{2}\left(B_{2}\right) \oplus B_{2}
$$

The second summand comes from the Universal Coefficient Theorem and the fact that $\operatorname{Tor}\left(B_{2}, \mathbf{F}_{2}\right) \cong B_{2}$. Let $\{f, g\} \in H_{2}\left(\mathcal{K}_{\mathrm{g}, 1}, \mathbf{F}_{2}\right)$ be an abelian cycle. Then it is straightforward to verify (see e.g. Lemma 5.3 of $[\mathrm{Sa}]$ ) that

$$
\sigma_{*}(\{f, g\})=(\sigma(f) \wedge \sigma(g), 0) \in \wedge^{2} B_{2} \oplus B_{2}
$$

Our strategy to prove that an abelian cycle in $H_{2}\left(\mathcal{K}_{\mathrm{g}, 1}, \mathbf{Z}\right)$ is nontrivial will be to show that its image under $\sigma_{*}$ is nontrivial.

Remark. It is impossible to detect classes corresponding to the $B_{2}$ summand of $H_{2}\left(B_{2}, \mathbf{F}_{2}\right)$ using this method, since an abelian cycle arises from a map of $\mathbf{Z}^{2}$ into $\mathcal{K}_{\mathrm{g}, 1}$ and any such class in $H_{2}\left(\mathcal{K}_{\mathrm{g}, 1}, \mathbf{F}_{2}\right)$ must vanish in the Tor term of the Universal Coefficient Theorem. For this reason we will write $\wedge^{2} B_{2} \subset H_{2}\left(B_{2}, \mathbf{F}_{2}\right)$ and will refer to the element $(x \wedge y, 0) \in$ $H_{2}\left(B_{2}, \mathbf{F}_{2}\right)=\wedge^{2} B_{2} \oplus B_{2}$ simply as $x \wedge y \in \wedge^{2} B_{2}$. Thus the formula (9) becomes simply

$$
\sigma_{*}(\{f, g\})=\sigma(f) \wedge \sigma(g) \in H_{2}\left(B_{2}, \mathbf{F}_{2}\right)
$$

\subsection{Calculations with $\sigma_{*}$}

Our strategy for proving Theorem 1 is twofold. First, we will use the induced map on homology $\sigma_{*}$ together with the formula (9) to hit basis elements of $\wedge^{2} B_{2}$ directly with abelian cycles. Secondly, we will use the fact that $\sigma$ is a $\operatorname{Mod}_{\mathrm{g}, 1^{-}}$equivariant map (and hence so is $\sigma_{*}$ ) to increase our efficiency. Thus we will compute orbits of basis elements of $\wedge^{2} B_{2}$ and note that hitting one element of an orbit with $\sigma_{*}$ tells us that every basis element in the orbit is also in the image of $\sigma_{*}$. 
A basis for $\wedge^{\mathbf{2}} \boldsymbol{B}_{2}$. Recall that a basis $\mathcal{B}$ for $B_{2}$ consists of the following $d=2 g^{2}+g+1$ Boolean monomials:

$$
\mathcal{B}=\left\{1, \bar{a}_{i}, \bar{b}_{i}, \bar{a}_{i} \bar{b}_{j}, \bar{a}_{i} \bar{a}_{j}(i \neq j), \bar{b}_{i} \bar{b}_{j}(i \neq j)\right\}
$$

where the $a_{i}, b_{j}$ are the fixed symplectic basis for $H_{1}\left(S_{g, 1}, \mathbf{F}_{2}\right)$ shown in Figure 1. Thus a basis for $\wedge^{2} B_{2}$ is given by

$$
\left(\begin{array}{l}
d \\
2
\end{array}\right)=2 g^{4}+2 g^{3}+\frac{3}{2} g^{2}+\frac{g}{2}
$$

elements of the form $m_{1} \wedge m_{2}$, where $m_{1}, m_{2}$ are distinct monomials in $\mathcal{B}$.

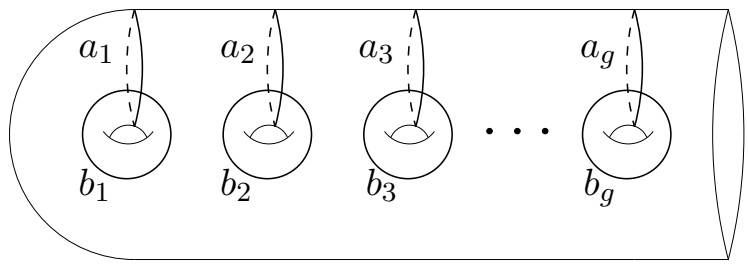

Figure 1: A fixed symplectic basis for $H_{1}\left(S_{g, 1}, \mathbf{F}_{2}\right)$

When trying to describe a basis for $\wedge^{2} B_{2}$, it is convenient to organize elements according to distinct patterns of the indices occurring in the monomials involved, which also have some topological significance. We particularly wish to distinguish elements of the form

$$
\bar{a}_{i} \bar{x} \wedge \bar{b}_{i} \bar{y}
$$

where $x$ and $y$ are arbitrary basis elements of $H$. We refer to such a basis element of $\wedge^{2} B_{2}$ as index-matched. We allow that possibility that $x=a_{i}$ or $y=b_{i}$, so that either or both factors of the wedge product could be linear.

We are now able to state our main homology calculation.

Theorem 3. For $g \geq 4$, the image of the map $\sigma_{*}: H_{2}\left(\mathcal{K}_{\mathrm{g}, 1}, \mathbf{F}_{2}\right) \rightarrow \wedge^{2} B_{2}$ contains the subspace $W$ spanned by all basis elements which are not index-matched. This space has dimension equal to a polynomial $p(g)=16 g^{4}+O\left(g^{3}\right)$.

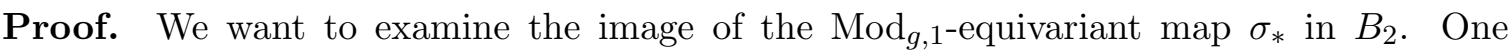
approach would be to calculate equivalence classes of basis elements of $B_{2}$ under the full action of $\operatorname{Mod}_{g, 1}$. However, it turns out to be more efficient to consider only the action of two particularly simple $\operatorname{Mod}_{g, 1}$-maps on the $\mathbf{F}_{2}$-vector space $B_{2}$ :

$$
\begin{gathered}
\bar{a}_{i} \mapsto \bar{b}_{i} \mapsto \bar{a}_{i} \\
\bar{a}_{i} \mapsto \bar{a}_{j} ; \quad \bar{b}_{i} \mapsto \bar{b}_{j}
\end{gathered}
$$

Elements not mentioned in the above maps are understood to be fixed. Recall that $W$ is the subspace of $B_{2}$ spanned by all basis elements which are not index-matched. What we 
do now is to calculate equivalence classes of basis elements of $W$ under the two maps given by (11) and (12). There are 11 equivalence classes of basis elements of $W$. We refer to these 11 equivalence classes as partial Sp-orbits. They are:

For all $i, j, k, l=1, \ldots, g, \quad i \neq j \neq k \neq l$ :

$$
\begin{aligned}
\mathrm{I} & =\left\{\bar{a}_{i} \bar{b}_{i} \wedge \bar{a}_{j} \bar{b}_{j}\right\} \\
\mathrm{II} & =\left\{\bar{a}_{i} \bar{b}_{i} \wedge \bar{a}_{j} \bar{b}_{k}, \bar{a}_{i} \bar{b}_{i} \wedge \bar{a}_{j} \bar{a}_{k}, \bar{a}_{i} \bar{b}_{i} \wedge \bar{b}_{j} \bar{b}_{k}\right\} \\
\mathrm{III} & =\left\{\bar{a}_{i} \bar{a}_{j} \wedge \bar{a}_{k} \bar{a}_{l}, \bar{a}_{i} \bar{a}_{j} \wedge \bar{a}_{k} \bar{b}_{l}, \bar{a}_{i} \bar{a}_{j} \wedge \bar{b}_{k} \bar{b}_{l}, \bar{a}_{i} \bar{b}_{j} \wedge \bar{a}_{k} \bar{b}_{l}, \bar{a}_{i} \bar{b}_{j} \wedge \bar{a}_{k} \bar{b}_{l}, \bar{b}_{i} \bar{b}_{j} \wedge \bar{b}_{k} \bar{b}_{l}\right\} \\
\mathrm{IV} & =\left\{\bar{a}_{i} \bar{a}_{j} \wedge \bar{a}_{i} \bar{a}_{k}, \bar{a}_{i} \bar{a}_{j} \wedge \bar{a}_{i} \bar{b}_{k}, \bar{a}_{i} \bar{b}_{j} \wedge \bar{a}_{i} \bar{b}_{k}, \bar{a}_{i} \bar{b}_{j} \wedge \bar{a}_{k} \bar{b}_{j}, \bar{a}_{i} \bar{b}_{j} \wedge \bar{b}_{j} \bar{b}_{k}, \bar{b}_{i} \bar{b}_{j} \wedge \bar{b}_{i} \bar{b}_{k}\right\} \\
\mathrm{V} & =\left\{\bar{a}_{i} \wedge \bar{a}_{j} \bar{b}_{j}, \bar{b}_{i} \wedge \bar{a}_{j} \bar{b}_{j}\right\} \\
\mathrm{VI} & =\left\{\bar{a}_{i} \wedge \bar{a}_{i} \bar{a}_{j}, \bar{a}_{i} \wedge \bar{a}_{i} \bar{b}_{j}, \bar{b}_{i} \wedge \bar{a}_{j} \bar{b}_{i}, \bar{b}_{i} \wedge \bar{b}_{i} \bar{b}_{j}\right\} \\
\mathrm{VII} & =\left\{\bar{a}_{i} \wedge \bar{a}_{j} \bar{a}_{k}, \bar{a}_{i} \wedge \bar{a}_{j} \bar{b}_{k}, \bar{a}_{i} \wedge \bar{b}_{j} \bar{b}_{k}, \bar{b}_{i} \wedge \bar{a}_{j} \bar{a}_{k}, \bar{b}_{i} \wedge \bar{a}_{j} \bar{b}_{k}, \bar{b}_{i} \wedge \bar{b}_{j} \bar{b}_{k}\right\} \\
\mathrm{VIII} & =\left\{1 \wedge \bar{a}_{i} \bar{b}_{i}\right\} \\
\mathrm{IX} & =\left\{1 \wedge \bar{a}_{i} \bar{a}_{j}, 1 \wedge \bar{a}_{i} \bar{b}_{j}, 1 \wedge \bar{b}_{i} \bar{b}_{j}\right\} \\
\mathrm{X} & =\left\{\bar{a}_{i} \wedge \bar{a}_{j}, \bar{a}_{i} \wedge \bar{b}_{j}, \bar{b}_{i} \wedge \bar{b}_{j}\right\} \\
\mathrm{XI} & =\left\{1 \wedge \bar{a}_{i}, 1 \wedge \bar{b}_{i}\right\}
\end{aligned}
$$

Direct calculations. We will work our way through the above-listed orbits one at a time, building on our previous calculations as we go. We will give a few calculations explicitly in order to give the reader the idea of how to proceed. For the remaining cases, we will give the readers the required abelian cycles and leave the calculations as an exercise.

We begin by noting a fact which will simplify our calculations. Let $\alpha, \beta$ be two simple closed curves on a surface such that $i(\alpha, \beta)=1$. Then the boundary $\gamma$ of a regular neighborhood $N$ of $\alpha \cup \beta$ is a genus 1 separating curve. Further, the two curves $\alpha$ and $\beta$ form a symplectic basis for $H_{1}\left(N, \mathbf{F}_{2}\right)$, and hence $\sigma\left(T_{\gamma}\right)=\bar{\alpha} \bar{\beta}$ (in general we will not distinguish between a curve and its homology class).

We call the pair $\alpha, \beta$ a spine for the genus 1 separating curve $\gamma$. We will also abuse terminology and refer to the spine of the map $T_{\gamma}$ and the spine of the subsurface bounded by $\gamma$. Thus when trying to "hit" a specific basis element of $\wedge^{2} B_{2}$, we will in general look first for spines which give the desired monomials. Clearly, disjoint spines correspond to disjoint separating curves and hence to commuting twists in $\mathcal{K}_{\mathrm{g}, 1}$.

Partial orbit I. Referring to Figure 2 , let $\gamma_{i}, \gamma_{j}$ be the two separating curves corresponding to the two 'spines' shown on the $i^{t h}$ and $j^{t h}$ holes, respectively. Then $\left\{T_{\gamma_{i}}, T_{\gamma_{j}}\right\}$ is an abelian cycle in $H_{2}\left(\mathcal{K}_{\mathrm{g}, 1}, \mathbf{F}_{2}\right)$, and using (6) and (9) gives

$$
\begin{aligned}
\sigma_{2}\left(\left\{T_{\gamma_{i}}, T_{\gamma_{j}}\right\}\right) & =\sigma\left(T_{\gamma_{i}}\right) \wedge \sigma\left(T_{\gamma_{j}}\right) \\
& =\bar{a}_{i} \bar{b}_{i} \wedge \bar{a}_{j} \bar{b}_{j}
\end{aligned}
$$




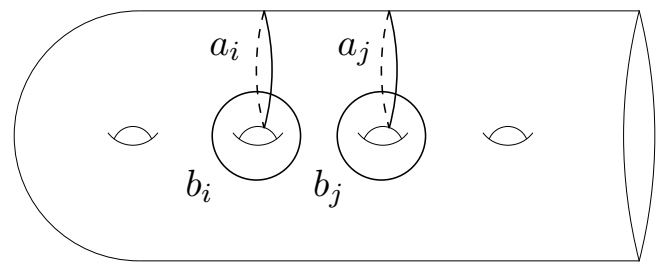

Figure 2: The spines of two bounding curves corresponding to an abelian cycle in $H_{2}\left(\mathcal{K}_{\mathrm{g}, 1}, \mathbf{F}_{2}\right)$ which maps to an element of Partial orbit I.

Partial orbit II. Let $\gamma, \delta$ denote the two separating curves corresponding to the spines shown in Figure 3; let the holes being 'used' in the picture be the $i^{\text {th }}, j^{\text {th }}$, and $k^{\text {th }}$ holes, respectively. Then $\left\{T_{\gamma}, T_{\delta}\right\}$ is an abelian cycle, and

$$
\begin{aligned}
\sigma_{2}\left(\left\{T_{\gamma}, T_{\delta}\right\}\right) & =\sigma\left(T_{\gamma}\right) \wedge \sigma\left(T_{\delta}\right) \\
& =\bar{a}_{i} \bar{b}_{i} \wedge \bar{a}_{j} \bar{c} \\
& =\bar{a}_{i} \bar{b}_{i} \wedge \bar{a}_{j}\left(\overline{b_{j}+a_{k}}\right) \\
& =\bar{a}_{i} \bar{b}_{i} \wedge \bar{a}_{j}\left(\bar{b}_{j}+\bar{a}_{k}+b_{j} \cdot a_{k}\right) \\
& =\bar{a}_{i} \bar{b}_{i} \wedge\left(\bar{a}_{j} \bar{b}_{j}+\bar{a}_{j} \bar{a}_{k}\right) \\
& =\bar{a}_{i} \bar{b}_{i} \wedge \bar{a}_{j} \bar{b}_{j}+\bar{a}_{i} \bar{b}_{i} \wedge \bar{a}_{j} \bar{a}_{k}
\end{aligned}
$$

Since the first term on the right-hand side of the last equality is already in the image of $\sigma_{2}$ (Partial orbit I), so is the second term.

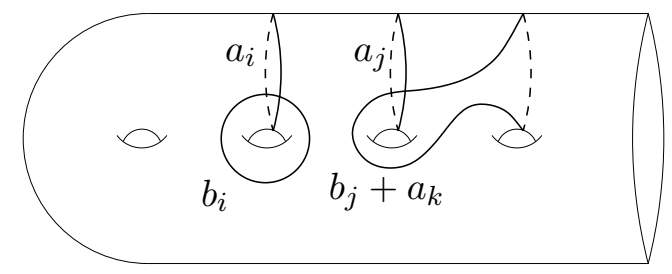

Figure 3: The spines of two bounding curves corresponding to an abelian cycle mapping to Partial orbit II.

Remark. Note that there exists $h \in \operatorname{Mod}_{g, 1}$ taking the spine (hence the corresponding abelian cycle) illustrated in Figure 2 to that illustrated in Figure 3. Hence these abelian cycles lie in the same Sp-orbit, namely (partial) orbit I. The calculation just made exhibits an element from (partial) orbit II as a difference of these two cycles.

We next give an example showing how to hit a linear term.

Partial orbit V. Let $\gamma, \delta$ denote the two separating curves corresponding to the spines shown in Figure 4; let the holes being 'used' in the picture be the $i^{t h}, j^{t h}$, and $k^{\text {th }}$ holes, 


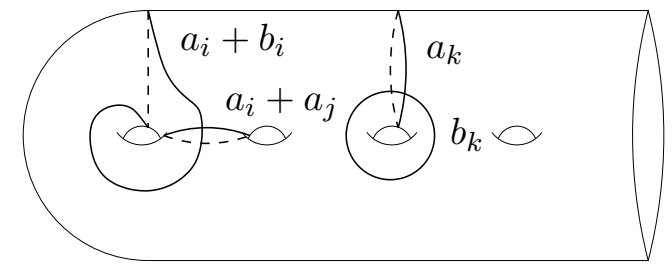

Figure 4: The spines of two bounding curves corresponding to an abelian cycle mapping to partial orbit V.

respectively. Then $\left\{T_{\gamma}, T_{\delta}\right\}$ is an abelian cycle, and

$$
\begin{aligned}
\sigma_{2}\left(\left\{T_{\gamma}, T_{\delta}\right\}\right) & =\sigma\left(T_{\gamma}\right) \wedge \sigma\left(T_{\delta}\right) \\
& =\left(\overline{a_{i}+b_{i}}\right)\left(\overline{a_{i}+a_{j}}\right) \wedge \bar{a}_{k} \bar{b}_{k} \\
& =\left(\bar{a}_{i}+\bar{b}_{i}+1\right)\left(\bar{a}_{i}+\bar{a}_{j}\right) \wedge \bar{a}_{k} \bar{b}_{k} \\
& =\left(\bar{a}_{i} \bar{b}_{i}+\bar{a}_{i} \bar{a}_{j}+\bar{a}_{j} \bar{b}_{i}+\bar{a}_{j}\right) \wedge \bar{a}_{k} \bar{b}_{k} \\
& =\bar{a}_{i} \bar{b}_{i} \wedge \bar{a}_{k} \bar{b}_{k}+\bar{a}_{i} \bar{a}_{j} \wedge \bar{a}_{k} \bar{b}_{k}+\bar{a}_{j} \bar{b}_{i} \wedge \bar{a}_{k} \bar{b}_{k}+\bar{a}_{j} \wedge \bar{a}_{k} \bar{b}_{k}
\end{aligned}
$$

Since the first three terms on the right-hand side of the last equality come from partial orbits I and II, it follows that partial orbit V is also contained in the image of $\sigma_{*}$.

The remaining calculations required for the proof of Theorem 3 are similar in flavor, although lengthier; we defer them to the Appendix, where we present figures indicating the necessary abelian cycles corresponding to each of the remaining (partial) orbits listed above.

Remark. In many of our calculations, such as the three given above, we do not use the hypothesis that $g \geq 4$; lower bounds may thus be obtained for $g<4$.

Cokernel. Let IM denote the subspace of $\wedge^{2} B_{2}$ spanned by index-matched basis elements. If one considers an index-matched element of the form $\bar{a}_{i} x \wedge \bar{b}_{i} y$ where $x$ and $y$ are distinct basis element with distinct indices, then it is clear that the dimension of IM is a polynomial in $g$ with highest degree term $4 g^{3}$. However, it turns out that "most" of IM is actually contained in the image of $\sigma_{*}$.

Proposition 4. The cokernel of $\sigma_{*}: H_{2}\left(\mathcal{K}_{\mathrm{g}, 1}, \mathbf{F}_{2}\right) \rightarrow H_{2}\left(B_{2}, \mathbf{F}_{2}\right)$ has dimension at most a polynomial with highest degree term $4 g^{2}$.

Proof. Theorem 3 above tells us that the dimension of the cokernel of $\sigma_{*}$ is at most $\operatorname{dim}(\mathrm{IM})$. Index-matched elements of the type $\bar{a}_{i} x \wedge \bar{b}_{i} y$, where $x$ and $y$ are distinct basis element with distinct indices, are the only index-matched basis elements contributing a cubic term to $\operatorname{dim}(\mathrm{IM})$. The subspace spanned by such elements has dimension $g(2 g-2)(2 g-3)$

Figure 5 below shows two abelian cycles. The first, when taken together with Theorem 3 , shows that the image of $\sigma_{*}$ contains any sum of the form

$$
\bar{a}_{i} \bar{b}_{i} \wedge \bar{a}_{i} \bar{b}_{j}+\bar{a}_{j} \bar{b}_{j} \wedge \bar{a}_{i} \bar{b}_{j}
$$


The second, when combined with Theorem 3 and (13), and after an adjustment of indices, shows that any sum of the form

$$
a_{i} b_{j} \wedge b_{i} a_{k}+a_{l} b_{j} \wedge b_{l} a_{k}
$$

(where all distinct indices are assumed to be not equal) is contained in the image of $\sigma_{*}$. The

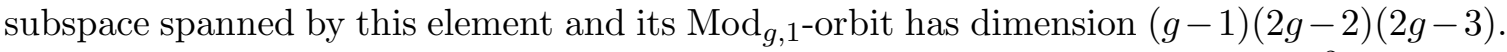
It follows that index-matched elements of the above type contribute at most $4 g^{2}-10 g+6$ to the dimension of the cokernel of $\sigma_{*}$.
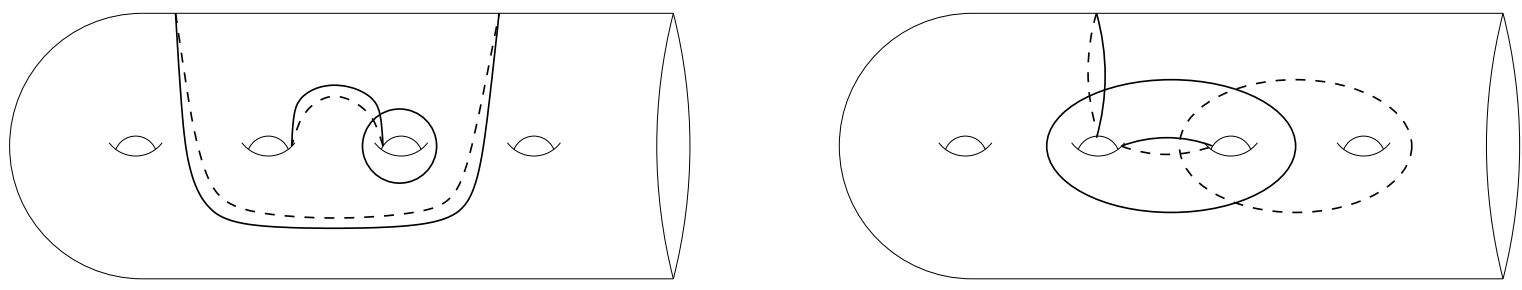

Figure 5: Two spines giving sums of pairs of index-matched elements.

Next, we consider index-matched basis elements whose orbits contribute a $g^{2}$-term to the dimension of IM. Calculations similar to the above show that the image of $\sigma_{*}$ also contains sums of these basis elements spanning a subspace whose dimension has the same quadratic term. Thus the remaining index-matched basis elements contribute at most a linear term to the dimension of the cokernel of $\sigma_{*}$. This completes the proof of the proposition.»

\section{Integral abelian cycles and the Casson-Morita algebra}

We next investigate the question of whether the mod 2 abelian cycles constructed in the previous section can be lifted to integral classes.

\subsection{The Casson-Morita algebra}

As described in $\S 2.1$ above, the $\mathbf{F}_{2}$-algebra $B_{3}$ is a kind of "universal receptor" for Rochlin invariants, or more precisely Birman-Craggs homomorphisms (see $\S 2.1$ above). We now explain how Morita lifted this setup to $\mathbf{Z}$ using the Casson invariant.

Casson invariant. The Casson invariant $\lambda(M)$ of a homology 3 -sphere $M$ is, roughly speaking, half the algebraic number of conjugacy classes of irreducible representations of $\pi_{1}(M)$ into $\mathrm{SU}(2)$. The invariant $\lambda$ is integer-valued, and is a lifting of Rochlin's $\mathbf{F}_{2}$-valued invariant, defined in Section 2.1, in the sense that $\lambda(M) \equiv \mu(M) \bmod 2$. See [AM] for a thorough exposition of the Casson invariant.

Let $h: \Sigma_{g} \rightarrow S^{3}$ be a Heegaard embedding, and let $f \in \mathcal{I}_{\mathrm{g}}$. Now split $S^{3}$ along $h\left(\Sigma_{g}\right)$ and reglue via the map $f$. By Meyer-Vietoris, the resulting 3-manifold $M(h, f)$ is a homology 3 -sphere. Just as described in Section 2.1 above for the Rochlin invariant, one can define a function $\lambda_{h}: \mathcal{I}_{\mathrm{g}} \rightarrow \mathbf{Z}$ given by $f \mapsto \lambda(M(h, f))$. 
While $\lambda_{h}$ is generally not a homomorphism, Morita [Mo1, Mo2] proved that the restriction $\lambda_{h}: \mathcal{K}_{\mathrm{g}} \rightarrow \mathbf{Z}$ is a homomorphism. He also showed that every homology 3 -sphere may be represented as $M_{\phi}$ for some $\phi \in \mathcal{K}_{\mathrm{g}}$. See [Mo1].

The Casson-Morita algebra. The method of combining all the homomorphisms $\lambda_{h}$ into one package is motivated by Casson's knot invariant. Let $K$ be a knot in a homology sphere $M$, and let $M_{K}$ denote the homology 3-sphere which results from doing $(1,1)$-surgery on $K$. Define $\lambda^{\prime}(K):=\lambda\left(M_{K}\right)-\lambda(M)$. Morita [Mo1, Mo2] has shown that if $c$ is a separating curve on $\Sigma_{g, 1}$ then

$$
\lambda_{h}\left(T_{c}\right)=-\lambda^{\prime}(h(c))
$$

Now consider the Seifert matrix $L=\left(l_{i j}\right)$ of the knot $K$, given with respect to some choice of symplectic basis for the homology of a Seifert surface for $K$. By definition, $l_{i j}$ is the linking number of the $i^{\text {th }}$ basis element with the positive push-off of the $j^{\text {th }}$ basis element. It turns out that $\lambda^{\prime}(K)$ can be expressed as a polynomial of degree 2 in the $l_{i j}$; this polynomial is given explicitly in [Mo1].

The Casson-Morita algebra $\mathcal{A}$ is then defined as follows. Let $\mathcal{A}$ be the commutative Z-algebra generated by the abstract symbols $l(u, v)$, where $u, v \in H_{1}(S)$, satisfying the relations:

1. $l(v, u)=l(u, v)+u \cdot v$

2. $l\left(n_{1} u_{1}+n_{2} u_{2}, v\right)=n_{1} l\left(u_{1}, v\right)+n_{2} l\left(u_{2}, v\right)$ for $m, n \in \mathbf{Z}$

For each Heegaard embedding $h: S \rightarrow S^{3}$, the algebra $\mathcal{A}$ has an evaluation homomorphism $\epsilon_{h}: \mathcal{A} \rightarrow \mathbf{Z}$ given by

$$
\epsilon_{h}(l(u, v))=l k\left(h_{*}(u), h_{*}(v)^{+}\right)
$$

\subsection{Morita's homomorphism $\rho$}

The Morita homomorphism $\rho: \mathcal{K}_{\mathrm{g}, 1} \rightarrow \mathcal{A}$, introduced in [Mo1], is defined as follows. Given a generator $T_{c} \in \mathcal{K}_{\mathrm{g}, 1}$, such that the curve $c$ bounds a subsurface $S^{\prime}$ with symplectic homology basis $A_{1}, \ldots, A_{g}, B_{1}, \ldots, B_{g}$, we set

$$
\begin{aligned}
\rho\left(T_{c}\right)= & -\sum_{i=1}^{g\left(S^{\prime}\right)}\left[l\left(A_{i}, A_{i}\right) l\left(B_{i}, B_{i}\right)-l\left(A_{i}, B_{i}\right) l\left(B_{i}, A_{i}\right)\right] \\
& -2 \sum_{i<j \leq g\left(S^{\prime}\right)}\left[l\left(A_{i}, A_{j}\right) l\left(B_{i}, B_{j}\right)-l\left(A_{i}, B_{j}\right) l\left(A_{j}, B_{i}\right)\right]
\end{aligned}
$$

Morita [Mo1] proved this assignment extends to a homomorphism on all of $\mathcal{K}_{\mathrm{g}, 1}$. The map $\rho$ plays a similar role with regard to the Casson invariant to that of the BCJ map with regard to the Rochlin invariant; we will describe the relationship of $\rho$ with the Casson invariant explicitly in Proposition 6 below. In fact, Morita's homomorphism $\rho$ is actually a lift of the BCJ homomorphism, just as the the Casson invariant is a lift of the Rochlin invariant. 
Proposition 5. There exists a homomorphism $\mu: \mathcal{A} \rightarrow B_{2}$ such that the following diagram commutes:

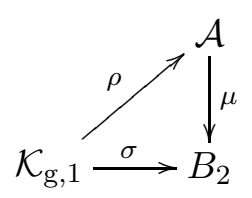

We would like to point out that the "reduction homomorphism" $\mu$ must respect the relations in the algebra $\mathcal{A}$, and so takes a more complicated form than a simple "reduction $\bmod 2 "$.

Proof. Before making any assignments for the value of $\mu$ on any of the generating symbols $l(a, b)$, we make a few motivational observations. First, note that the image of the second term of Equation 14 under any homomorphism to a 2-group must necessarily be 0 . Also, we claim that the first defining relation in $\mathcal{A}$ gives that the image of the second part of the first term of Equation 14 must also map to 0, since we have:

$$
\begin{aligned}
l\left(A_{i}, B_{i}\right) l\left(B_{i}, A_{i}\right) & =l\left(A_{i}, B_{i}\right)\left[l\left(A_{i}, B_{i}\right)+A_{i} \cdot B_{i}\right] \\
& =l\left(A_{i}, B_{i}\right)^{2}+\left(A_{i} \cdot B_{i}\right) l\left(A_{i}, B_{i}\right) \\
& =l\left(A_{i}, B_{i}\right)^{2}+l\left(A_{i}, B_{i}\right)
\end{aligned}
$$

The square-free condition in $B_{2}$ now implies the claim.

Thus we can focus our attention on the image of $l\left(A_{i}, A_{i}\right)$. Now, the obvious assignment which makes the diagram commutative is

$$
\mu\left(l\left(A_{i}, A_{i}\right)\right)=\bar{A}_{i} \in B_{2}
$$

The question becomes: how does one define the map $\mu$ on the entire algebra $\mathcal{A}$ so as to achieve the desired result?

Let $a_{1}, \ldots, a_{g}, b_{1}, \ldots, b_{g}$ be the fixed symplectic basis for $H_{1}(S)$. Then the defining relations show that symbols of the form $l(x, y)$, where $x, y \in\left\{a_{1}, \ldots, a_{g}, b_{1}, \ldots, b_{g}\right\}$, suffice to generate $\mathcal{A}$. We now set, for all $1 \leq i, j \leq g$ :

$$
\begin{aligned}
& \mu\left(l\left(a_{i}, a_{i}\right)\right)=\bar{a}_{i}, \quad \mu\left(l\left(b_{i}, b_{i}\right)\right)=\bar{b}_{i} \\
& \mu\left(l\left(a_{i}, b_{j}\right)\right)=0 \\
& \mu\left(l\left(b_{j}, a_{i}\right)\right)=\mu\left(l\left(a_{i}, a_{j}\right)\right)=\mu\left(l\left(b_{i}, b_{j}\right)\right)=\delta_{i j}
\end{aligned}
$$

A straightforward check shows that these assignments satisfy the defining relations of $\mathcal{A}$, and thus by extending linearly we get a well-defined homomorphism $\mu: \mathcal{A} \rightarrow B_{2}$. Further, it is also straightforward (although a bit tedious) to check that this definition on the fixed basis also gives $\mu(l(u, u))=\bar{u}$ for any $u \in H_{1}(S)$, and thus the diagram commutes. $\diamond$

We are now ready to prove the following. 
Proposition 6. The following diagram commutes:

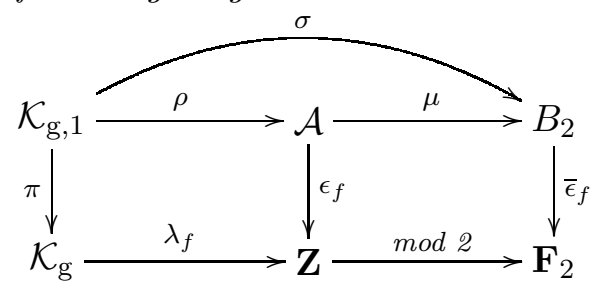

Proof. The fact that the left-hand square commutes is Theorem 2.2 of [Mo2]. The commutativity of the upper 'triangle' is Proposition 5 above. It remains to deal with the right-hand square.

Let $\omega_{f}$ denote the mod 2 self-linking form on $H_{1}(S)$ induced by the Heegaard embedding $f: \Sigma_{g, 1} \rightarrow M$. Recalling the definition of elements of $B_{2}$ from Section 2.1, we have the following natural analog of an evaluation map on $B_{2}$ :

$$
\begin{aligned}
\bar{\epsilon}_{f}(\bar{u}) & =\bar{u}\left(\omega_{f}\right) \\
& =l k\left(f_{*}(u), f_{*}(u)^{+}\right) \bmod 2
\end{aligned}
$$

It is now straightforward to check the commutativity of the right-hand square; again, it suffices to check on the generators $l\left(a_{i}, b_{j}\right)$, etc. This finishes the proof of the proposition. $\diamond$

\subsection{Lifting abelian cycles}

We next describe how the $\mathbf{F}_{2}$-classes constructed as in Section 3.2 can be lifted to integral classes. Define $p: H_{2}\left(\mathcal{K}_{\mathrm{g}, 1}, \mathbf{Z}\right) \rightarrow H_{2}\left(\mathcal{K}_{\mathrm{g}, 1}, \mathbf{F}_{2}\right)$ to be the composition

$$
H_{2}\left(\mathcal{K}_{\mathrm{g}, 1}, \mathbf{Z}\right) \rightarrow H_{2}\left(\mathcal{K}_{\mathrm{g}, 1}, \mathbf{Z}\right) \otimes \mathbf{F}_{2} \rightarrow H_{2}\left(\mathcal{K}_{\mathrm{g}, 1}, \mathbf{F}_{2}\right)
$$

where the first map is given by $f \mapsto f \otimes 1$ and the second map is the injection given by the Universal Coefficients Theorem. The analogous map $q: H_{2}\left(B_{2}, \mathbf{Z}\right) \rightarrow H_{2}\left(B_{2}, \mathbf{F}_{2}\right)$ is just the injection arising from the Universal Coefficients Theorem. Proposition 6 directly implies the following.

Proposition 7. The following diagram commutes.

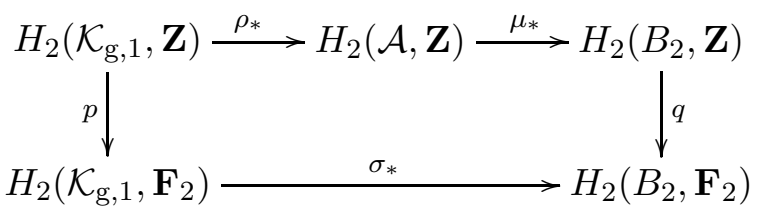

Note that, by construction, the map $p$ sends an abelian cycle $\{f, g\}$ in $H_{2}\left(\mathcal{K}_{\mathrm{g}, 1}, \mathbf{Z}\right)$ to the 'same' abelian cycle in $H_{2}\left(\mathcal{K}_{\mathrm{g}, 1}, \mathbf{F}_{2}\right)$. The proposition tells us that if $\xi \in H_{2}\left(\mathcal{K}_{\mathrm{g}, 1}, \mathbf{Z}\right)$ and $\sigma_{*} p(\xi) \neq 0$, then $\xi \neq 0$ as well. In other words, each of the $\mathbf{F}_{2}$ classes arising from abelian cycles which were constructed in Section 3.2 and in the Appendix also lift to integral classes. Thus we also obtain lower bounds on the rank of $H_{2}\left(\mathcal{K}_{\mathrm{g}, 1}, \mathbf{Z}\right)$ as a corollary to Theorem 3 . 
Corollary 8. The rank of $\mathrm{H}_{2}\left(\mathcal{K}_{\mathrm{g}, 1}, \mathbf{Z}\right)$ is at least $16 g^{4}+O\left(g^{3}\right)$.

Remark. In light of the relationship between the maps $\rho$ and $\sigma$, it is natural to ask whether we could gain any information about $H_{2}\left(\mathcal{K}_{\mathrm{g}, 1}, \mathbf{Z}\right)$ by working directly with integer coefficients, rather than with $\mathbf{F}_{2}$. However, note that the $\mathbf{Z}$-rank of $H_{2}(\mathcal{A}, \mathbf{Z})$ is precisely equal to the $\mathbf{F}_{2}$-dimension of $H_{2}\left(B_{2}, \mathbf{F}_{2}\right)$. Thus we lost no information by working with $\sigma$ and with $\mathbf{F}_{2}$ coefficients. Morever, the calculations required for the proof of Theorem 3 were greatly simplified by working mod 2 .

\section{Cohomology}

The following commutative diagram summarizes the various relationships between $H_{*}\left(\mathcal{K}_{\mathrm{g}, 1}, \mathbf{F}_{2}\right)$ and $H^{*}\left(\mathcal{K}_{\mathrm{g}, 1}, \mathbf{F}_{2}\right)$, the induced maps $\sigma_{*}$ and $\sigma^{*}$, and their images.

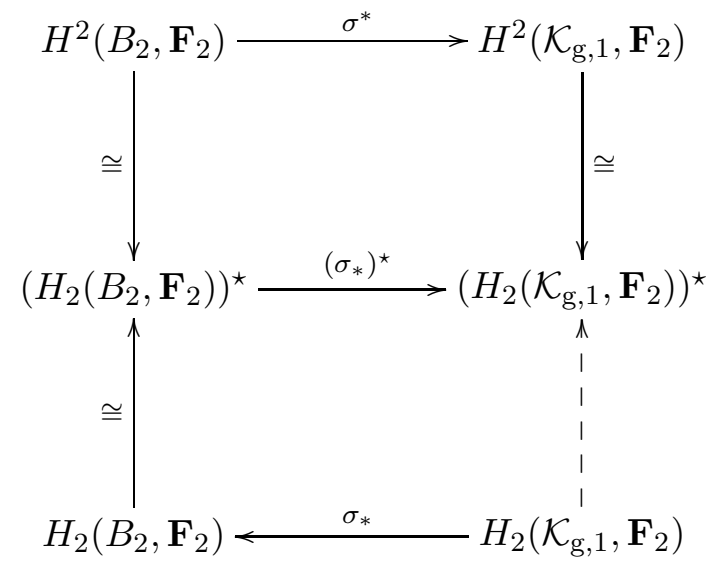

Here $\sigma_{*}^{\star}$ denotes the dual of the induced map $\sigma_{*}$ on homology. The commutativity of the top square comes directly from the Universal Coefficient Theorem for cohomology. We emphasize that we do not necessarily get an isomorphism between $H_{2}\left(\mathcal{K}_{\mathrm{g}, 1}, \mathbf{F}_{2}\right)$ and $\left(H_{2}\left(\mathcal{K}_{\mathrm{g}, 1}, \mathbf{F}_{2}\right)\right)^{\star}$ since it is not known whether or not $H_{2}\left(\mathcal{K}_{\mathrm{g}, 1}, \mathbf{F}_{2}\right)$ is finite dimensional.

Given $\xi_{*} \in H_{2}\left(B_{2}, \mathbf{F}_{2}\right)$, we let $\xi^{*}$ denote its isomorphic image in $H^{2}\left(B_{2}, \mathbf{F}_{2}\right)$. It now follows easily from the diagram that

$$
\xi_{*} \in \operatorname{Im} \sigma_{*} \Rightarrow \xi^{*} \notin \operatorname{ker} \sigma^{*}
$$

Thus Theorem 3 implies Theorem 1. In fact, one can determine from the nontriviality of the abelian cycle $\{f, g\} \in H_{2}\left(B_{2}, \mathbf{F}_{2}\right)$ the nontriviality of the image under $\sigma^{*}$ of a corresponding cup product of elements in $H^{1}\left(B_{2}, \mathbf{F}_{2}\right)$.

Further classes. As previously noted, all abelian cycles constructed in $H_{2}\left(\mathcal{K}_{\mathrm{g}, 1}, \mathbf{F}_{2}\right)$ survive in $H_{2}\left(\mathcal{I}_{\mathrm{g}}, \mathbf{F}_{2}\right)$. Of course, one could also construct more classes by utilizing Johnson's formula for bounding pair maps in addition to his formula for twists about separating curves. This combinatorial challenge has been successfully undertaken by Vijay Ravikumar [Ra], who is able to prove the following: 
Proposition 9 (Ravikumar). The image under $\sigma_{*}$ of the full Torelli group contains a subspace of dimension $64 g^{6}+$ lower-order terms.

This $g^{6}$ lower bound is the best order-of-magnitude one can hope for using the method of abelian cycles.

\section{Appendix: Calculations for the proof of Theorem 3}

In this appendix we list figures indicating the abelian cycles necessary to complete the proof of Theorem 3. In each case, the pictures show two spines corresponding to an abelian cycle in $H_{2}\left(\mathcal{K}_{\mathrm{g}}, \mathbf{F}_{2}\right)$, as in the proof of Theorem 3 above.

We also remind the reader that a given figure labelled by a particular Sp-orbit may not give an element of that orbit exactly, but rather may correspond to a sum of an element in that orbit together with basis elements already known to be in the image of $\sigma_{*}$.

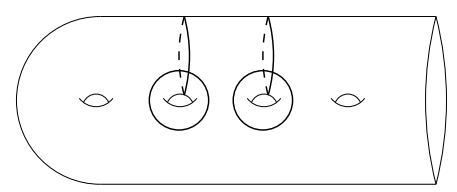

Orbit I

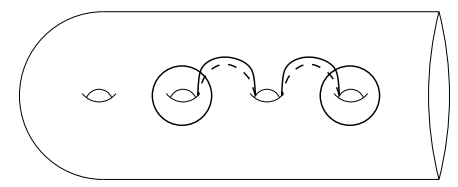

Orbit IV

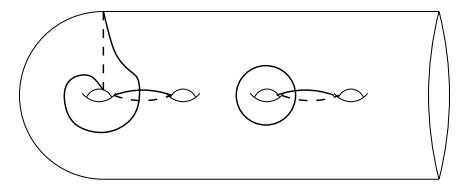

Orbit VII

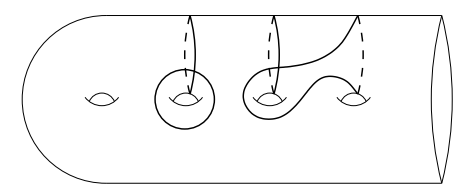

Orbit II

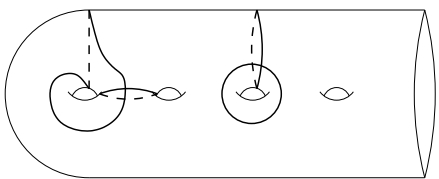

Orbit V

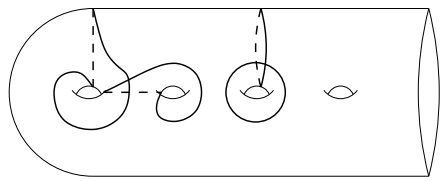

Orbit VII

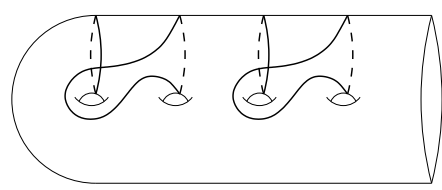

Orbit III

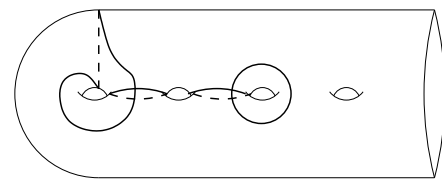

Orbit VI

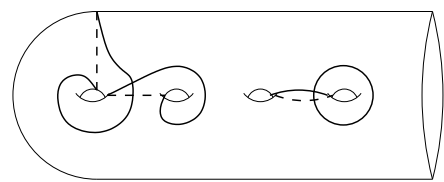

Orbit IX

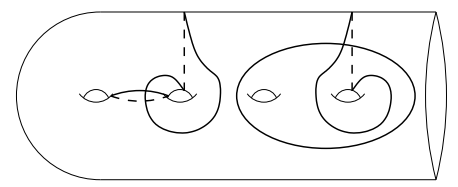

Orbit X

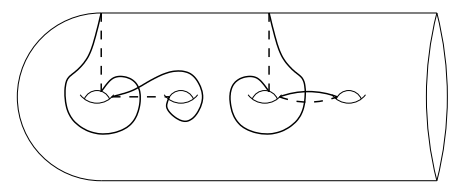

Orbit XI 
We conclude by listing some abelian cycles which indicate how to find more sums of indexmatched basis elements.
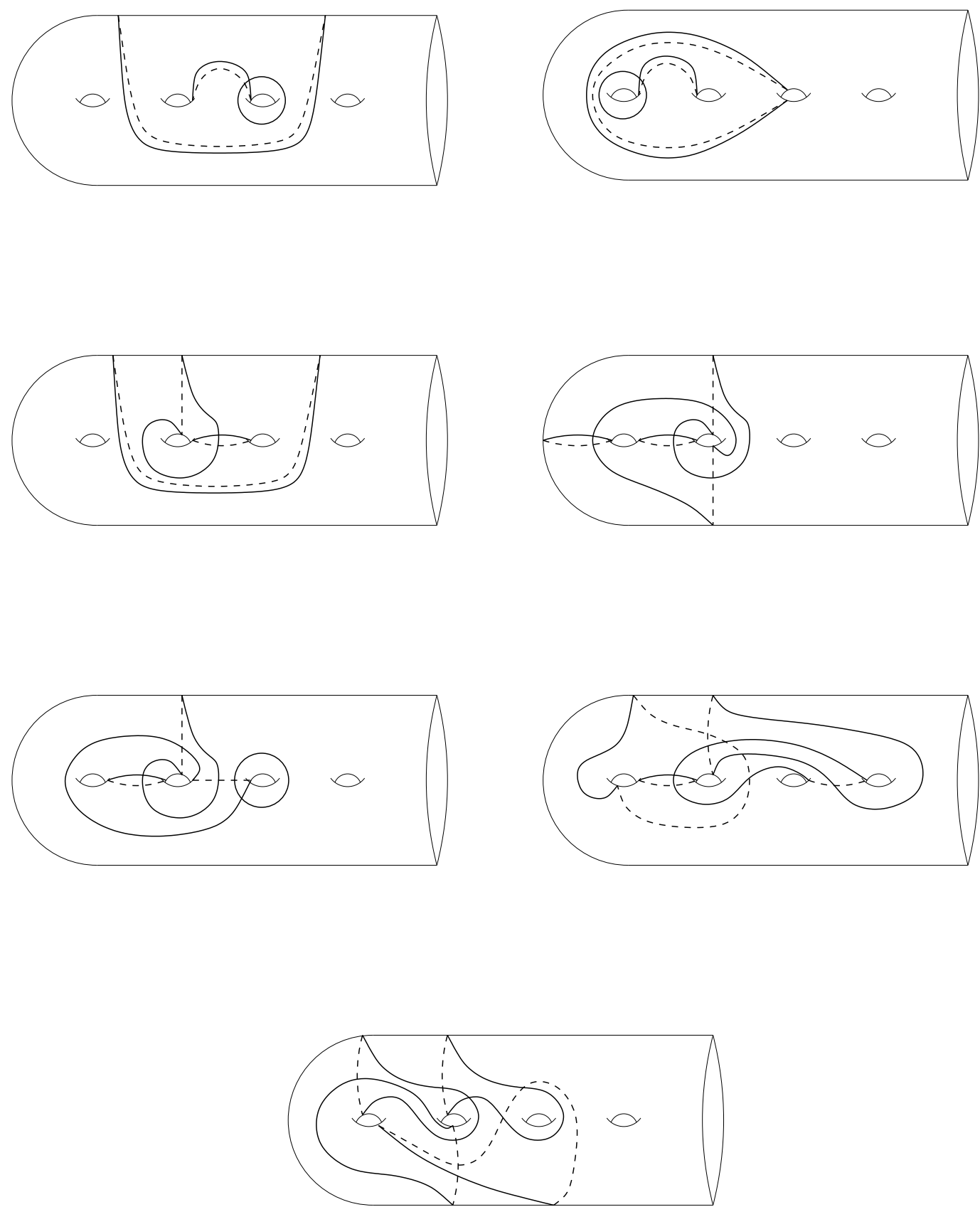


\section{References}

[Ak] T. Akita, Homological infiniteness of Torelli groups, Topology, Vol. 40 (2001), no. 2, 213-221.

[AM] S. Akbulut and J. McCarthy, Casson's invariant for oriented homology 3-spheres: an exposition, Mathematical Notes 36, Princeton University Press, 1990.

[BC] J. Birman and R. Craggs, The $\mu$-invariant of 3-manifolds and certain structural properties of the group of homeomorphisms of a closed, oriented 2-manifold, Trans. of the AMS, Vol. 237 (1978), p. 283-309.

[BF] D. Biss and B. Farb, $\mathcal{K}_{g}$ is not finitely generated, Invent. Math., Vol. 163, pp. 213-226 (2006).

[Br] K. Brown, Cohomology of groups, Graduate Texts in Mathematics, Vol. 87, Springer, 1982.

[Ga] S. Galatius, Mod $p$ homology of the stable mapping class group, Topology, Vol. 43 (2004), pp.1105-1132.

[GS] R. Gompf and A. Stipsicz, 4-Manifolds and Kirby Calculus, AMS Graduate Studies in Mathematics Vol. 20, 1999.

[Hai] R. Hain, Infinitesimal presentations of the Torelli groups, JAMS, Vol. 10, No. 3 (1997), pp. 597-651.

[Ha] J. Harer, The second homology group of the mapping class group of an orientable surface, Invent. Math., Vol. 72 (1983), pp. 221-239.

[Hat] A. Hatcher, Algebraic Topology, Cambridge University Press, 2002.

[Jo1] D. Johnson, Quadratic forms and the Birman-Craggs homomorphisms, Trans. of the $A M S$, Vol. 261, No. 1 (1980), pp. 423-422.

[Jo2] D. Johnson, The structure of the Torelli group I: A finite set of generators for $\mathcal{I}$, Annals of Math., Vol. 118, No. 3 (1983), pp. 423-422.

[Jo3] D. Johnson, The structure of the Torelli group II: A characterization of the group generated by twists on bounding curves, Topology, Vol. 24, No. 2 (1985), pp. 113-126.

[Jo4] D. Johnson, The structure of the Torelli group III: the abelianization of $\mathcal{I}_{\mathrm{g}}$, Topology, Vol. 24, No. 2 (1985), pp. 127-144.

[Jo5] D. Johnson, A survey of the Torelli group, Contemporary Mathematics, Vol. 20 (1983), pp. 165-179.

[Jo6] D. Johnson, An abelian quotient of the mapping class group $\mathcal{I}_{\mathrm{g}_{g}}$, Math. Ann., Vol. 249 (1980), pp. 225-242.

[MW] I. Madsen and M. Weiss, The stable moduli space of Riemann surfaces: Mumford's conjecture, preprint (2002), arXiv:math.AT/0212321.

[MM] D. McCullough and A. Miller, The genus 2 Torelli group is not finitely generated, Topology Appl., Vol. 22 (1986), pp. 43-49. 
[Me] G. Mess, The Torelli groups for genus 2 and 3 surfaces, Topology, Vol. 31, No. 4 (1992), pp. $775-790$.

[Mo1] S. Morita, Casson's invariant for homology 3-spheres and characteristic classes of surface bundles I, Topology, Vol. 28, No. 3 (1989), pp. 305-323.

[Mo2] S. Morita, On the structure of the Torelli group and the Casson invariant, Topology, Vol 30, No. 4 (1991), pp. 603-621.

[Ra] V. Ravikumar, private communication.

[Sa] T. Sakasai, The Johnson homomorphism and the third rational cohomology group of the Torelli group, UTMS preprint 2003-21, (http://kyokan.ms.utokyo.ac.jp/users/preprint/ps/2003-21.ps).

[vdB] B. van den Berg, On the abelianization of the Torelli group, $\mathrm{Ph}$. D. Thesis, Universiteit Utrecht (2003), (https://dspace.library.uu.nl:8443/retrieve/25176/inhoud.htm).

Tara E. Brendle:

Dept. of Mathematics, Louisiana State University

Lockett Hall

Baton Rouge, LA 70803

E-mail: brendle@math.lsu.edu

Benson Farb:

Dept. of Mathematics, University of Chicago

5734 University Ave.

Chicago, Il 60637

E-mail: farb@math.uchicago.edu 